\title{
The Effect of a High Fat Meal on Cerebral Vascular Function
}

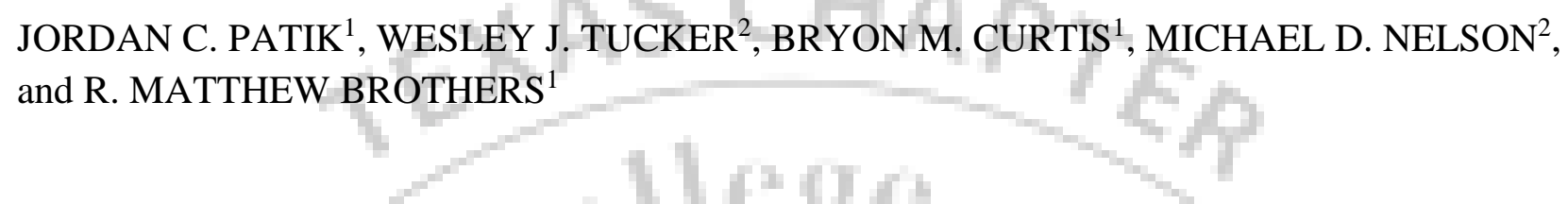

1Integrative Vascular Physiology Laboratory; Department of Kinesiology; The University of Texas at Arlington; Arlington, TX

2Applied Physiology and Advanced Imaging Laboratory; Department of Kinesiology;

The University of Texas at Arlington; Arlington, TX

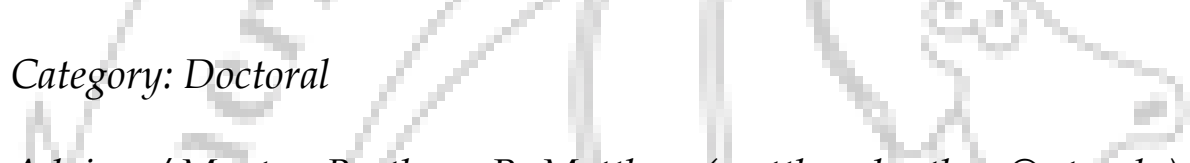

Advisor / Mentor: Brothers, R. Matthew (matthew.brothers@uta.edu)

ABSTRACT

It is well known that a single high fat meal (HFM) causes a robust and transient elevation in serum triglycerides (TG). This elevation in serum TG is a primary contributor to the post-prandial attenuation of peripheral vascular endothelial function, as assessed by flow-mediated dilation in the brachial artery. Whether a similar impairment in vascular reactivity can be observed in the cerebral circulation remains unknown, and was the focus of this investigation. PURPOSE: To test the hypothesis that cerebral vascular function is impaired following a HFM. METHODS: End-tidal carbon dioxide partial pressure (PETCO2), middle cerebral artery blood velocity (MCAVmean), calculated cerebral vascular conductance index (CVCI; MCAVmean/mean arterial pressure) and cerebral vasodilator response to rebreathing induced hypercapnia (\% increase in CVC from baseline at common maximal $\triangle \mathrm{PETCO} 2$ ) were assessed in 6 healthy young men ( $27 \pm 5$ years). Measures were assessed during fasted baseline and again at 2 and $4 \mathrm{~h}$ post meal consumption (HFM day) or at a similar time point in the fasted state (TC day). The two visits were separated by 2-7 days and were conducted in a randomized order. Blood lipids were assessed at baseline and at the $2 \mathrm{~h}$ time point into each respective condition. RESULTS: As expected, consumption of the HFM significantly elevated serum TG concentrations relative to TC at $2 \mathrm{~h}$ (HFM: $101 \pm 38$ to $169 \pm 77 \mathrm{mg} / \mathrm{dl}$, TC: $107 \pm 32$ to $92 \pm 31 \mathrm{mg} / \mathrm{dl}, \mathrm{P}=0.007)$. However, the HFM had no effect of cerebral vasodilator capacity during rebreathing induced hypercapnia. The maximal increase in \% CVC achieved at the highest common $\triangle$ PETCO2 during all conditions within each subject was unchanged during $2 \mathrm{hr}$ and $4 \mathrm{hr}$ post HFM or TC (condition $x$ time interaction: $\mathrm{P}=0.96$ ). Similarly, the slope of the change in $\% \mathrm{CVC}$ per change in $\triangle \mathrm{PETCO} 2$ was unaffected by HFM across time $(\mathrm{P}=0.49)$. CONCLUSION: Contrary to our hypothesis, and unlike the peripheral vasculature, our preliminary data suggest that the cerebral circulation appears to be protected from the acute negative effects of a high fat meal.

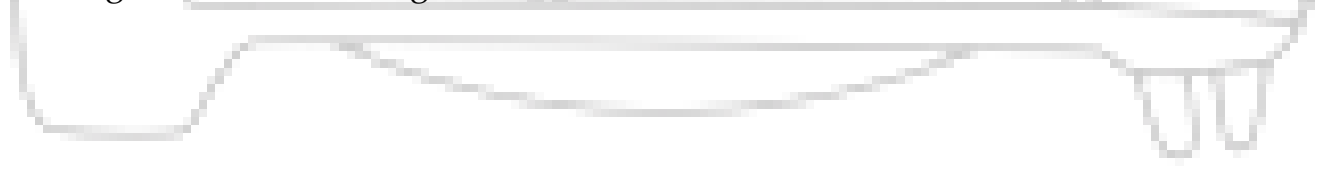

